Preprint 1/2006

\title{
Least Squares Importance Sampling for Monte Carlo Security Pricing
}

\author{
Luca Capriott: \\ Global Modelling and Analytics Group, Investment Banking Division, Credit Suisse Group \\ One Cabot Square, London, E14 4QJ, United Kingdom
}

(Dated: October 30, 2018)

\begin{abstract}
We describe a simple Importance Sampling strategy for Monte Carlo simulations based on a least squares optimization procedure. With several numerical examples, we show that such Least Squares Importance Sampling (LSIS) provides efficiency gains comparable to the state of the art techniques, when the latter are known to perform well. However, in contrast to traditional approaches, LSIS is not limited to the determination of the optimal mean of a Gaussian sampling distribution. As a result, it outperforms other methods when the ability to adjust higher moments of the sampling distribution, or to deal with non-Gaussian or multi-modal densities, is critical to achieve variance reductions.
\end{abstract}

Keywords: Monte Carlo Simulations, Variance Reduction Techniques, Importance Sampling, Derivatives Pricing.

\section{INTRODUCTION}

The impressive development of the securities markets has generated in the last few years a steady demand for more and more structured financial products. At the same time, the level of sophistication and complexity of the pricing models employed by investment firms has dramatically increased, in a continuous search for a possible edge against competitors. As a result, an increasing fraction of the models employed in practice is too complex to be treated by analytic or deterministic numerical methods (trees or partial differential equations), and Monte Carlo simulation becomes more often than ever the only computationally feasible means of pricing and hedging.

Although generally easy to implement, Monte Carlo simulations are infamous for being slow. In fact, being stochastic in nature, their outcome is always affected by a statistical error, that can be generally reduced to the desired level of accuracy by iterating the calculation for long enough time. This comes with a high computational cost as such statistical uncertainties, all things being equal, are inversely proportional to the square root of the number of statistically independent samples. Hence, in order to reduce the error by a factor of 10 one has to spend 100 times as much computer time. For this reason, to be used on a trading floor, Monte Carlo simulations often require to be run on large parallel computers with a high financial cost in terms of hardware, infrastructure, and software development.

Motivated by this very practical necessity, several approaches to speed up Monte Carlo calculations, such as Antithetic Variables, Control Variates, and Importance Sampling, have been proposed over the last few years [1]. These techniques aim to reduce the variance per Monte Carlo observation so that a given level of accuracy can be obtained with a smaller number of iterations. In general, this can be done by exploiting some information known

*Electronic address: luca.capriotti@credit-suisse.com. a priori on the structure of the problem at hand, like a symmetry property of the Brownian paths (Antithetic Variables), the value of a closely related security (Control Variates), or the form of the statistical distribution of the random samples (Importance Sampling). Antithetic Variables and Control Variates are the most commonly used variance reduction techniques, mainly because of the simplicity of their implementation, and the fact that they can be accommodated in an existing Monte Carlo calculator with a small effort. However, their effectiveness varies largely across applications, and is sometimes rather limited [1].

On the other hand, Importance Sampling techniques, although potentially more powerful, have not been employed much in professional contexts until recently. This is mainly because such techniques generally involve a bigger implementation effort, and they are also less straightforward to include in a general Monte Carlo framework. Moreover, when used improperly, Importance Sampling can increase the variance of the Monte Carlo estimators, thus making its integration in an automated environment more problematic. Nonetheless, the potential efficiency gains at stake are so large that the interest in finding efficient Importance Sampling schemes is still very high.

The idea behind Importance Sampling is to reduce the statistical uncertainty of a Monte Carlo calculation by focusing on the most important sectors of the space from which the random samples are drawn. Such regions critically depend on both the random process simulated, and the structure of the security priced. For instance, for a deep out-of-the money Call option [2], the payoff sampled is zero for most of the iterations of a Monte Carlo simulation. Hence, simulating more samples with positive payoff reduces the variance. This can be done by changing the probability distribution from which the samples are drawn, and reweighing the payout function by the appropriate likelihood-ratio (Radon-Nikodyn derivative) in order to produce an unbiased result of the original problem [1].

Most of the work in Importance Sampling methods for security pricing has been done in a Gaussian setting 
$[3,4,5,6,6,18,9,10]$ such the one arising from the simulation of a diffusion process. In this framework, Importance Sampling is achieved by modifying the drift term of the simulated process in order to drive the Brownian paths towards the regions that are the most important for the evaluation of the security. For instance, for the Call option above, this can be obtained by increasing the drift term up to a certain optimal level [3, 4]. The different approaches proposed in the literature, essentially differ in the way in which such change of drift is found, and can be roughly divided into two families depending on the strategy adopted. The first strategy, common to the so-called adaptive Monte Carlo methods [5, 8, 9, 10], aims to determine the optimal drift through stochastic optimization techniques that typically involve an iterative algorithm. On the other hand, the second strategy, proposed in a remarkable paper by Glasserman, Heidelberger, and Shahabuddin (GHS) [6] , relies on a deterministic optimization procedure that can be applied for a specific class of payouts. This approach, although approximate, turns out to be very effective for several pricing problems, including the simulation of a single factor Heath-Jarrow-Morton model [7], and portfolio credit scenarios [11].

In this paper, we propose the Least Squares Importance Sampling (LSIS), as an effective and flexible variance reduction method for Monte Carlo security pricing. This approach, originally proposed in Physics for the optimization of quantum mechanical wave functions of correlated electrons [12], is applied here in a financial setting. In LSIS the determination of the optimal drift or more in general of the most important regions of the sample space - is formulated in terms of a least squares minimization. This technique can be easily implemented and included in an existing Monte Carlo code, and simply relies on a standard least square algorithm for which several optimized libraries are available. We will show that LSIS provides efficiency gains analogous to the ones of previously proposed methods when the latter are known to perform well. However, LSIS does not share some of their limitations, and its range of applications includes multi-modal problems and non-Gaussian sampling.

In the following Section, we begin by reviewing the main ideas behind Importance Sampling in a generic Monte Carlo framework. Then in Section III we specialize the discussion to the Gaussian setting, and we briefly review the recent adaptive strategies, and the GHS approach of Ref. [6]. The rationale of LSIS is introduced in Section IV together with the essential implementation details, and in Section V we present the results of our numerical experiments. Here we perform a systematic study of the variance reductions obtained by means of LSIS for a variety of test cases, including a comparison with recent Importance Sampling techniques. Finally, we draw our conclusions in Section VI.

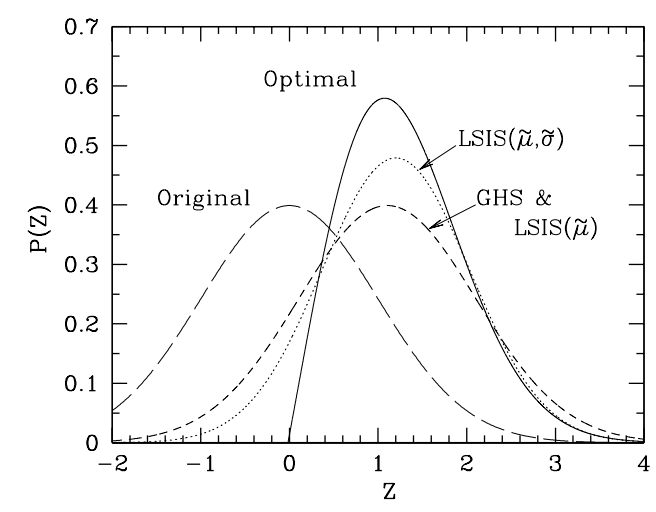

FIG. 1: Sampling probability density functions for a European Call option with $T=1, r=0.05, \sigma=0.3, X_{0}=K=50$ as obtained with LSIS [optimizing just the drift, $\operatorname{LSIS}(\tilde{\mu})$, and both the drift and the volatility, $\operatorname{LSIS}(\tilde{\mu}, \tilde{\sigma})]$, and the saddle point approximation (GHS). On this scale the results for $\operatorname{LSIS}(\tilde{\mu})$ and GHS are indistinguishable. The original (13) and the optimal (9) sampling densities are also shown for comparison.

\section{IMPORTANCE SAMPLING}

Let us consider the general problem of estimating the expectation value of a scalar function, $G(Z)$, depending on a $d$-dimensional real random vector $Z=\left(Z_{1}, \ldots, Z_{d}\right)$ with joint probability distribution $P(Z)$,

$$
V=E_{P}[G(Z)]=\int_{D} d Z G(Z) P(Z),
$$

where $D$ is the domain of possible values of the state variables $Z$ [19]. In a financial derivatives context, $G(Z)$ would typically represent the discounted payout of a certain security, and $P(Z)$ would be the risk neutral-probability measure of an arbitrage free market [13, 14, 15]. For instance, for the familiar Call option in the Black-Scholes framework [2] one has $d=1$, $P(Z)=(2 \pi)^{-1 / 2} \exp \left(-Z^{2} / 2\right)$ and

$$
G(Z)=e^{-r T}\left(X_{0} \exp \left[\left(r-\frac{\sigma^{2}}{2}\right) T+\sigma Z\right]-K\right)^{+}
$$

where $r$ is the risk-free interest rate, $\sigma$ is the volatility, $X_{0}$ and $K$ are respectively the spot and strike price, and $T$ the maturity of the option.

Whenever the dimension $d$ of the state variable $Z$ is large (say $d \gtrsim 4$ ) standard numerical quadrature approaches become highly inefficient, and Monte Carlo methods are the only feasible route for estimating expectation values of the form (11). To do so, one interprets Eq. (11) as a weighted average of the payout function $G(Z)$ over the possible configurations $Z$ with weights given by the probability distribution $P(Z)$. This immediately leads to the simplest (and crudest) Monte Carlo estimator which is obtained by averaging the payout function 


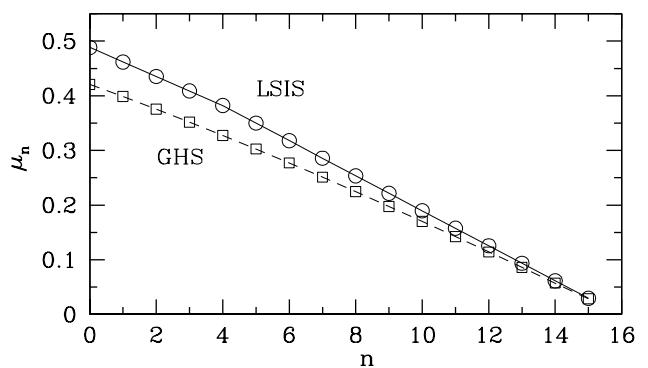

FIG. 2: Optimal drift vector as obtained with the LSIS and the GHS procedures for an arithmetic Asian Call option (15), $\Phi(\bar{X})=\left(\bar{X}-K^{+}\right)$, on a lognormal asset (14) for $M=16$ $\sigma=0.3, X_{0}=K=50, r=0.05$, and $T=1.0$.

over a sample of $N_{p}$ independent values of the random variable $Z$ generated according to the probability distribution $P(Z)$,

$$
V \simeq \bar{V}=\frac{1}{N_{p}} \sum_{i=1}^{N_{p}} G\left(Z_{i}\right) \quad Z_{i} \sim P(Z) .
$$

In particular, the central limit theorem [16] ensures that, for big enough samples, the values of the estimator $\bar{V}$ are normally distributed around the true value, and converge for $N_{p} \rightarrow \infty$ towards $V$ namely

$$
V \simeq \frac{1}{N_{p}} \sum_{i=1}^{N_{p}} G\left(Z_{i}\right) \pm \frac{\Sigma}{\sqrt{N_{p}}}
$$

where $\Sigma^{2}=E_{P}\left[G(x)^{2}\right]-E_{P}[G(x)]^{2}$ is the variance of the estimator and can be similarly approximated by

$$
\Sigma^{2} \simeq \frac{1}{N_{p}} \sum_{i=1}^{N_{p}}\left(G\left(Z_{i}\right)-\bar{V}\right)^{2}
$$

Although Eq. (41) ensures the convergence of the Monte Carlo estimator to the expectation value (1), its practical utility depends on the magnitude of the variance, $\Sigma^{2}$. Indeed, the square root convergence in (4), implies that the number of replications $N_{p}$ that are (asymptotically) necessary to achieve a given level of accuracy is proportional to the variance of the estimator [20]. Roughly speaking, such quantity is relatively small whenever the function $G(Z)$ is approximately constant over the region of values of $Z$ that is represented the most among the random samples, i.e., the region that contains most of the probability mass of $P(Z)$. This is generally not the case for most of the pricing problems encountered in practice, and the calculation of accurate estimates of the expectation value (1) may require large sample sizes $N_{p}$, thus becoming computationally demanding.

However, the choice of extracting the random variable $Z$ according to the probability distribution $P(Z)$, although natural, is by no means the only possible one.
Indeed, the Monte Carlo integration can be performed by sampling an arbitrary probability distribution $\tilde{P}(Z)$ provided that the integral is suitably reweighed. In fact, using the identity

$$
\int_{D} d Z G(Z) P(Z)=\int_{D} d Z \frac{G(Z) P(Z)}{\tilde{P}(Z)} \tilde{P}(Z),
$$

an alternative estimator of the expectation value (1) is readily found as

$$
V \simeq \tilde{V}=\frac{1}{N_{p}} \sum_{i=1}^{N_{p}} W\left(Z_{i}\right) G\left(Z_{i}\right) \quad Z_{i} \sim \tilde{P}(Z)
$$

with the weight function given by $W(Z)=P(Z) / \tilde{P}(Z)$. The variance of the new Monte Carlo estimator reads

$$
\tilde{\Sigma}^{2}=\int_{D} d Z(W(Z) G(Z)-V)^{2} \tilde{P}(Z)
$$

and critically depends on the choice of the sampling probability distribution $\tilde{P}(Z)$. For non-negative functions $G(Z)$, the optimal choice of $\tilde{P}(Z)$ is the one for which $\tilde{\Sigma}$ vanishes, namely:

$$
P_{\text {opt }}(Z)=\frac{1}{V} G(Z) P(Z) .
$$

In fact, the Monte Carlo estimator corresponding to such optimal sampling distribution reads

$$
\tilde{V} \simeq \frac{1}{N_{p}} \sum_{i=1}^{N_{p}} W\left(Z_{i}\right) G\left(Z_{i}\right)=\frac{1}{N_{p}} \sum_{i=1}^{N_{p}} V,
$$

leading to a constant value $V$ on each Monte Carlo replication, and resulting therefore in zero variance [21]. Unfortunately, such a choice is not really viable as the normalization constant, $V$, is the expectation value (1) we want to calculate in the first place.

\section{GAUSSIAN FRAMEWORK}

The Importance Sampling approaches proposed in the literature usually apply or are generally formulated within a Gaussian setting, i.e., in a context where the distribution $P(Z)$ defining the expectation value (11) is a $d$-dimensional standard normal distribution. For example, this is the case for a Monte Carlo simulation of a vector diffusive process of the form [1]

$$
d X(t)=\mu(X(t), t) d t+\sigma(X(t), t) d W_{t},
$$

e.g., for the calculation of the price of an option depending on the path followed by $X(t)$ within a certain time interval $[0, T]$. Here the precess $X(t)$ and the drift $\mu(X, t)$ are both $L$-dimensional real vectors, $W_{t}$ is a $N$ dimensional standard Brownian motion, and the volatility, $\sigma(X, t)$, is a $L \times N$ real matrix. 
TABLE I: Comparison between LSIS, the adaptive Robbins-Monro (RM) algorithm (as quoted in Ref. [10]), and the saddle point approach of Ref. [6] (GSH): price of a European Call option on a lognormal asset (14) for different values of the volatility $\sigma$, and of the strike price $K$. The variance reduction (VR) is defined in Eq. (26). The parameters used are $r=0.05, X_{0}=50$, $T=1.0$, and the number of simulated paths is 1,000,000 for Crude MC, LSIS and GHS, 50,000 for RM. Results for LSIS obtained by optimizing the drift only $[\operatorname{LSIS}(\tilde{\mu})]$, and both the drift and the volatility $[\operatorname{LSIS}(\tilde{\mu}, \tilde{\sigma})]$ are reported. The uncertainties on the least significative digits of the option prices, and variance reductions are reported in parentheses. Note that in Ref. [10] no error estimate was quoted for the RM results. However, from the ratio of the simulated paths, it is sensible to estimate the errors on the $\mathrm{RM}$ variance reductions as about $\sqrt{20} \simeq 4$ times larger than those quoted for $\operatorname{LSIS}(\tilde{\mu})$.

\begin{tabular}{|c|c|c|c|c|c|c|c|c|c|c|}
\hline \multirow[b]{2}{*}{$\sigma$} & \multirow[b]{2}{*}{$K$} & \multirow{2}{*}{$\begin{array}{l}\text { Crude MC } \\
\text { Price }\end{array}$} & \multicolumn{2}{|c|}{$\operatorname{LSIS}(\tilde{\mu})$} & \multicolumn{2}{|c|}{$\operatorname{LSIS}(\tilde{\mu}, \tilde{\sigma})$} & \multicolumn{2}{|c|}{ RM } & \multicolumn{2}{|c|}{ GHS } \\
\hline & & & Price & VR & Price & VR & Price & VR & Price & VR \\
\hline \multirow[t]{3}{*}{0.1} & 30 & $21.4633(50)$ & $21.46294(49)$ & $104(1)$ & $21.46296(12)$ & $1700(100)$ & 21.47 & $112(4)$ & $21.46294(50)$ & $100(1)$ \\
\hline & 50 & $3.4032(39)$ & $3.4019(14)$ & $7.8(1)$ & $3.4046(10)$ & $15(1)$ & 3.41 & $7.8(4)$ & $3.4018(14)$ & $7.8(1)$ \\
\hline & 60 & $0.2315(11)$ & $0.23112(19)$ & $33.5(5)$ & $0.23132(12)$ & $84(5)$ & 0.23 & $31(2)$ & $0.23126(19)$ & $33.5(5)$ \\
\hline \multirow[t]{3}{*}{0.3} & 30 & $21.598(15)$ & $21.5912(37)$ & $16.4(1)$ & $21.5984(21)$ & $51(1)$ & 21.63 & $16.8(4)$ & $21.5973(39)$ & $14.8(2)$ \\
\hline & 50 & $7.114(11)$ & $7.1169(35)$ & $9.9(5)$ & $7.1159(21)$ & $27(1)$ & 7.12 & $11(2)$ & $7.1146(35)$ & $9.9(1)$ \\
\hline & 60 & $3.4954(83)$ & $3.4514(21)$ & $15.6(1)$ & $3.4523(14)$ & $35(1)$ & 3.45 & $15.2(4)$ & $3.4508(22)$ & $14.2(1)$ \\
\hline
\end{tabular}

TABLE II: Same as Table @ for a European Put option.

\begin{tabular}{|c|c|c|c|c|c|c|c|c|c|c|}
\hline \multirow[b]{2}{*}{$\sigma$} & \multirow[b]{2}{*}{$K$} & \multirow{2}{*}{$\begin{array}{c}\text { Crude MC } \\
\text { Price }\end{array}$} & \multicolumn{2}{|c|}{$\overline{\operatorname{LSIS}(\tilde{\mu})}$} & \multicolumn{2}{|c|}{$\overline{\operatorname{LSIS}(\tilde{\mu}, \tilde{\sigma})}$} & \multicolumn{2}{|c|}{$\overline{\mathrm{RM}}$} & \multicolumn{2}{|l|}{ GHS } \\
\hline & & & Price & VR & Price & VR & Price & VR & Price & VR \\
\hline \multirow[t]{3}{*}{0.1} & 40 & $0.004235(98)$ & $0.0041650(47)$ & $435(6)$ & $0.0041616(41)$ & $571(9)$ & 0.0042 & $350(24)$ & $0.0041650(47)$ & $435(6)$ \\
\hline & 50 & $0.9636(19)$ & $0.96419(64)$ & $8.8(1)$ & $0.96436(38)$ & $25(2)$ & 0.97 & $9.6(4)$ & $0.96385(63)$ & $9.1(1)$ \\
\hline & 60 & $7.3052(46)$ & $7.3059(19)$ & $5.9(1)$ & $7.3056(11)$ & $17(1)$ & 7.31 & $6.3(4)$ & $7.3047(19)$ & $5.9(1)$ \\
\hline \multirow[t]{3}{*}{0.3} & 30 & $0.13397(83)$ & $0.13445(13)$ & $41(1)$ & $0.13448(10)$ & $69(2)$ & 0.13 & $38(4)$ & $0.013440(13)$ & $40.8(5)$ \\
\hline & 50 & $4.6794(65)$ & $4.6761(27)$ & $5.8(1)$ & $4.6767(16)$ & $16.5(5)$ & 4.68 & $6.2(4)$ & $4.6761(27)$ & $5.8(1)$ \\
\hline & 60 & $10.5203(97)$ & $10.5236(44)$ & $4.9(1)$ & $10.5266(26)$ & $13.9(2)$ & 10.54 & $4.8(4)$ & $10.5223(46)$ & $4.4(1)$ \\
\hline
\end{tabular}

Continuous time processes of the form (11) are typically simulated by sampling $X(t)$ on a discrete grid of points, $0=t_{0}<t_{1}<\ldots<t_{M}=T$, by means, for instance, of a Euler scheme [22]

$$
X_{i+1}=X_{i}+\mu\left(X_{i}, t\right) \Delta t_{i}+\sigma\left(X_{i}, t\right) \sqrt{\Delta t_{i}} Z_{i+1},
$$

where $X_{i}=X\left(t_{i}\right), \Delta t_{i}=t_{i+1}-t_{i}$, and $Z_{i+1}$ is a $N$ dimensional vector of independent standard normal variates. In this representation, each discretized path for the vector process $X(t)$ can be put into a one to one correspondence with a set of $d=N \times M$ independent standard normal variables $Z$. Hence, the original problem of evaluating the expectation value of a functional of the realized path of the process $X(t)$ can be formulated as in (11). More precisely, $G(Z)$ is given by the discretized payout functional, and the probability density is given by a $d$-dimensional standard normal distribution

$$
P(Z)=(2 \pi)^{-d / 2} e^{-Z^{2} / 2},
$$

where $Z^{2}=Z \cdot Z$.

As a prototypical example of exotic option pricing problem, treated as a test case for the most recently proposed Importance Sampling strategies [6, 8, 9, 10], in the following we will consider different arithmetic Asian style options under a Black-Scholes log-normal model [15]. In this case, the underlying asset can be simulated on the time grid relevant for the payout function by means of the exact recursion

$$
X_{i+1}=X_{i} \exp \left[\left(r-\sigma^{2} / 2\right) \Delta t_{i}+\sigma \sqrt{\Delta t_{i}} Z_{i+1}\right],
$$

where $r$ is the risk free interest rate, $\sigma$ is the constant $N \times L$ real volatility matrix, and $\sigma^{2}$ is a $L$-dimensional vector of components $\sigma_{k}^{2}=\sum_{j=1}^{N} \sigma_{k j}$. For an arithmetic Asian style claim, the discounted payout function is of the form

$$
G(Z)=e^{-r T} \Phi(\bar{X})
$$

where $\bar{X}=(1 / M) \sum_{i=1}^{M} X_{i}$, and $\Phi(\bar{X})$ is some function of $L$ variables. Clearly, European style options are recovered in the special case $M=1$.

\section{A. Gaussian Trial Distributions}

The simplest possible strategy for Importance Sampling in a Gaussian framework is to try to guide the sampled paths towards the most important regions of the configuration space (i.e., where the contribution of the integrand is the largest), by means of a change of the drift terms of the process (11) or (12). The corresponding trial probability density reads

$$
\tilde{P}_{\tilde{\mu}}(Z)=(2 \pi)^{-d / 2} e^{-(Z-\tilde{\mu})^{2} / 2},
$$


TABLE III: European Butterfly spread option (28) on a lognormal asset (14) for different values of the spot price: comparison between the saddle point approach of Ref. [6] (GHS), and LSIS with the optimization of both drift and volatility [LSIS( $\tilde{\mu}, \tilde{\sigma})$ ] The parameters used are $r=0.1, K_{1}=45, K_{2}=50, K_{3}=55, T=1.0, \sigma=0.3$, and the number of simulated paths is $1,000,000$.

\begin{tabular}{|c|c|c|c|c|c|}
\hline \multirow[b]{2}{*}{$X_{0}$} & \multirow{2}{*}{$\begin{array}{l}\text { Crude MC } \\
\text { Price }\end{array}$} & \multicolumn{2}{|c|}{ GHS } & \multicolumn{2}{|c|}{$\operatorname{LSIS}(\tilde{\mu}, \tilde{\sigma})$} \\
\hline & & Price & VR & Price & VR \\
\hline 30 & $0.15818(69)$ & $0.15732(33)$ & $4.4(8)$ & $0.157659(40)$ & $298(5)$ \\
\hline 40 & $0.4871(11)$ & $0.48666(98)$ & $1.26(1)$ & $0.48703(11)$ & $100(5)$ \\
\hline 50 & $0.6274(13)$ & $0.6274(13)$ & $1.00(1)$ & $0.62750(11)$ & $140(1)$ \\
\hline 60 & $0.5156(12)$ & $0.5157(10)$ & $1.44(1)$ & $0.515636(93)$ & $166(2)$ \\
\hline 70 & $0.32961(97)$ & $0.32875(67)$ & $2.10(1)$ & $0.329281(73)$ & $177(2)$ \\
\hline
\end{tabular}

TABLE IV: Comparison between LSIS and the saddle point approach of Ref. [6] (GSH): price of Asian options (15) on a lognormal asset (14) for different values of the volatility $\sigma$, the strike $K$, and of the number of observation dates $M$. The parameters used are $r=0.05, X_{0}=50, T=1.0$, and the number of simulated paths is $1,000,000$.

\begin{tabular}{|c|c|c|c|c|c|c|c|}
\hline \multirow[b]{2}{*}{$M$} & \multirow[b]{2}{*}{$\sigma$} & \multirow[b]{2}{*}{$K$} & \multirow{2}{*}{$\begin{array}{l}\text { Crude MC } \\
\text { Price }\end{array}$} & \multicolumn{2}{|c|}{ LSIS } & \multicolumn{2}{|c|}{ GHS } \\
\hline & & & & Price & VR & Price & VR \\
\hline \multirow[t]{3}{*}{16} & 0.1 & 45 & $6.0565(29)$ & $6.05522(88)$ & $10.86(5)$ & $6.05537(89)$ & $10.62(5)$ \\
\hline & & 50 & $1.9198(22)$ & $1.91994(80)$ & $7.56(5)$ & $1.91914(83)$ & $7.03(5)$ \\
\hline & & 55 & $0.20272(74)$ & $0.20235(16)$ & $21.4(2)$ & $0.20237(16)$ & $21.4(2)$ \\
\hline \multirow[t]{3}{*}{16} & 0.3 & 45 & $7.1545(77)$ & $7.1531(26)$ & $8.8(1)$ & $7.1529(27)$ & $8.13(1)$ \\
\hline & & 50 & $4.1730(63)$ & $4.1714(20)$ & $9.9(1)$ & $4.1712(21)$ & $9.0(1)$ \\
\hline & & 55 & $2.2135(48)$ & $2.2115(13)$ & $13.6(7)$ & $2.2116(14)$ & $11.8(7)$ \\
\hline \multirow[t]{3}{*}{64} & 0.1 & 45 & $5.9967(28)$ & $5.99510(87)$ & $10.4(5)$ & $5.99500(85)$ & $10.9(5)$ \\
\hline & & 50 & $1.8467(21)$ & $1.84522(78)$ & $7.2(3)$ & $1.84525(81)$ & $6.7(3)$ \\
\hline & & 55 & $0.17519(67)$ & $0.17448(14)$ & $23(2)$ & $0.17443(14)$ & $23(1)$ \\
\hline \multirow[t]{3}{*}{64} & 0.3 & 45 & $7.0257(75)$ & $7.0204(25)$ & $9.0(2)$ & $7.0204(26)$ & $8.3(2)$ \\
\hline & & 50 & $4.0271(61)$ & $4.0222(19)$ & $10.3(5)$ & $4.0220(20)$ & $9.3(5)$ \\
\hline & & 55 & $2.0849(46)$ & $2.0794(13)$ & $12.5(5)$ & $2.0794(13)$ & $12.5(5)$ \\
\hline
\end{tabular}

where $\tilde{\mu}$ is a $d$-dimensional vector, and the weight function, as also expected from the Girsamov theorem [14], is

$$
W_{\tilde{\mu}}(Z)=\exp \left[-\tilde{\mu} \cdot Z+\tilde{\mu}^{2} / 2\right] .
$$

A variety of approaches for the determination of the drift vector $\tilde{\mu}$ minimizing the variance of the estimator (8) has been recently proposed in the literature $[5,6,6,6,9,10]$. These can be roughly classified into two families depending on the strategy adopted. The first strategy, common to the so-called adaptive Monte Carlo methods, aims to determine the optimal drift vector though a stochastic minimization of the variance. Such minimization differs in details in the various methods but always involves an iterative procedure, to be performed in a preliminary Monte Carlo simulation.

In particular, $\mathrm{Su}$ and $\mathrm{Fu}$ [8, 9], building upon previous work by Vazquez-Abad and Dufresne [5], used a gradient-based stochastic approximation, dubbed infinitesimal perturbation analysis, in order to estimate the optimal uniform shift of the drift for the diffusion (12), minimizing the variance of the estimator (8). In the notation of this Section, this translates in working with a trial density of the form (16) where the drift vector $\tilde{\mu}$ has components all equal to a single optimization parameter. The improvement of this method with respect to the one of Ref. [5], is that the minimization is carried out under the original probability measure (as we also do for LSIS), while in the latter the minimization was formulated under the trial probability measure. As a result, the stochastic minimization applies also for non differentiable payout, thus making the approach more general. The application of this technique to partial average Asian options in a Black-Scholes market, and to caplets under the Cox-Ingersoll-Ross model provides significative variance reductions [8, 9].

Along similar ideas, Arouna [10] has recently proposed a different stochastic optimization method for the determination of the optimal sampling density (16). Here, in contrast to the previous approach, all the components of the drift vector are independently optimized. The method relies on a truncated version of the RobbinsMonro algorithm that is shown to converge asymptotically to the optimal drift, and to provide an effective variance reduction in a variety of cases. However, as remarked by the same author, a critical aspect of the practical implementation of the Robbins-Monro algorithm is that it depends on the size of the iterative step. Hence, a particular care needs to be taken in order for the algorithm to be efficient [23].

On the other hand, the second strategy, proposed by Glasserman, Heidelberger, and Shahabuddin (GHS) [6], 
TABLE V: Importance Sampling plus Stratification. Comparison between LSIS, and the GHS approach of Ref. [6] for the Asian Option of Table IV

\begin{tabular}{|c|c|c|c|c|c|c|}
\hline \multirow[b]{2}{*}{$M$} & \multirow[b]{2}{*}{$\sigma$} & \multirow[b]{2}{*}{$K$} & \multicolumn{2}{|c|}{ LSIS+ SS } & \multicolumn{2}{|c|}{$\mathrm{GHS}+\mathrm{SS}$} \\
\hline & & & Price & VR & Price & VR \\
\hline \multirow[t]{3}{*}{16} & 0.3 & 45 & $7.15284(25)$ & $950(20)$ & $7.15266(24)$ & $1030(10)$ \\
\hline & & 50 & $4.17122(18)$ & $1225(15)$ & $4.17118(18)$ & $1225(30)$ \\
\hline & & 55 & $2.21183(11)$ & $1900(100)$ & $2.21183(11)$ & $1900(50)$ \\
\hline \multirow[t]{3}{*}{64} & 0.3 & 45 & $7.02075(23)$ & $1060(30)$ & $7.02076(23)$ & $1060(30)$ \\
\hline & & 50 & $4.02251(17)$ & $1290(30)$ & $4.02250(17)$ & $1290(30)$ \\
\hline & & 55 & $2.07967(13)$ & $1320(100)$ & $2.07965(12)$ & $1470(100)$ \\
\hline
\end{tabular}

relies on a saddle point approximation to minimize the variance of the estimator (8), or equivalently of its second moment (in the original measure)

$$
m_{2}(\tilde{\mu})=\int_{D} d Z W_{\tilde{\mu}}(Z) G(Z)^{2} P(Z) .
$$

In fact, if the payout function $G(Z)$ is positive definite, by defining $F(Z)=\log G(Z)$ one can approximate Eq. (18) with the zero-order saddle point expansion

$$
\begin{aligned}
& (2 \pi)^{-d / 2} \int_{D} d Z \exp \left[2 F(Z)-\tilde{\mu} \cdot Z+\tilde{\mu}^{2} / 2-Z^{2} / 2\right] \\
\simeq & C \exp \left[\max _{Z \in D}\left(2 F(Z)-\tilde{\mu} \cdot Z+\tilde{\mu}^{2} / 2-Z^{2} / 2\right)\right],
\end{aligned}
$$

where $C$ is a constant. As a result, within this approximation, the problem of determining the optimal change of drift boils down to finding the vector $\mu$ such that

$$
\max _{Z \in D}\left(2 F(Z)-\tilde{\mu} \cdot Z+\tilde{\mu}^{2} / 2-Z^{2} / 2\right)
$$

is minimum. It is easy to show that this is obtained by choosing $\tilde{\mu}^{\star}=Z^{\star}$ where $Z^{\star}$ is the point that solves the optimization problem

$$
\max \left(F(Z)-Z^{2} / 2\right),
$$

or equivalently, for which the payout times the original distribution, $G(Z) P(Z)$, is maximum, i.e., $Z^{\star}$ corresponds to the maximum of the optimal sampling density, Eq. (9). The simplest interpretation of the saddle point approach is therefore that it approximates the zero variance distribution by means of a normal density with the same mode and variance.

The saddle point approach can be expected to be particularly effective in reducing the variance of the Monte Carlo estimator whenever the log payout function $F(Z)$ is close to be linear in the portion of the configuration space where most of the probability mass of $P(Z)$ lays. Moreover, GHS have also shown that stratifying [1] the $d$-dimensional random vector $Z$ along the optimal $\mu$, provides a spectacular variance reduction in certain cases [6, 7]. However, whenever the optimal sampling probability (9) cannot be accurately represented by a single Gaussian with the same mode and variance, the saddle point approximation is less beneficial. In particular, as it will be also illustrated in Sec. $\nabla$ this approach turns out to be less effective whenever the structure of the payout function $G(Z)$ is such that the optimal sampling distribution (9) has a width which is very different from the one of the original distribution, or is multi-modal.

In the following Section we describe an alternative least squares strategy that is straightforward to implement and flexible enough to be applied in a generic Monte Carlo setting. Indeed, the Least Squares Importance Sampling (LSIS) is not limited to the determination of the optimal change of drift in a Gaussian model. Instead, it can be applied to any Monte Carlo simulation provided that a reasonable guess of the optimal sampling density is available. For this reason, in the next Section we will momentarily leave the Gaussian framework, and we will describe the rationale of LSIS in a more general setting.

\section{LEAST SQUARES IMPORTANCE SAMPLING}

A practical approach to the search of an effective Importance Sampling distribution can be formulated in terms of a non-linear optimization problem. To this purpose, let us consider a family of trial probability densities, $\tilde{P}_{\theta}(Z)$, depending on a set of $N_{\theta}$ real parameters $\theta=\left(\theta_{1}, \theta_{2}, \ldots, \theta_{N_{\theta}}\right)$. The variance of the estimator corresponding to $\tilde{P}_{\theta}(Z)$, Eq. (8), can be written in terms of the original probability distribution $P(Z)$ as

$$
\tilde{\Sigma}_{\theta}^{2}=E_{P}\left[W_{\theta}(Z) G^{2}(Z)\right]-E_{P}[G(Z)]^{2},
$$

with $W_{\theta}(Z)=P(Z) / \tilde{P}_{\theta}(Z)$. Hence, the optimal Importance Sampling distribution within the family $\tilde{P}_{\theta}(Z)$ is the one for which the latter quantity, or equivalently the second moment (18) or

$$
E_{P}\left[W_{\theta}(Z) G^{2}(Z)\right]
$$

is minimum. The crucial observation is that the Monte Carlo estimator of this quantity,

$$
m_{2}(\theta) \simeq \frac{1}{N_{p}^{\prime}} \sum_{i=1}^{N_{p}^{\prime}}\left(W_{\theta}\left(Z_{i}\right)^{1 / 2} G\left(Z_{i}\right)\right)^{2} \quad Z_{i} \sim P(Z)
$$


can be interpreted as a non-linear least squares fit of a set of $N_{p}^{\prime}$ data points $\left(x_{i}, y_{i}\right)$ with a function $y=f_{\theta}(x)$ parameterized by $\theta$, with the correspondence $y_{i} \rightarrow 0$, $x_{i} \rightarrow Z_{i}$, and $f_{\theta}(x) \rightarrow W_{\theta}(Z)^{1 / 2} G(Z)$. The latter is a standard problem of statistical analysis that can be tackled with a variety of robust and easily accessible numerical algorithms, as the so-called Levenberg-Marquardt method [17].

Alternatively, to improve the numerical stability of the least-squares procedure, it is convenient in some situations to minimize, instead of (22), the pseudo-variance

$$
\begin{aligned}
S_{2}(\theta)= & E_{P}\left[\left(W_{\theta}(Z)^{1 / 2} G(Z)-V_{T}\right)^{2}\right] \\
& \simeq \frac{1}{N_{p}^{\prime}} \sum_{i=1}^{N_{p}^{\prime}}\left(W_{\theta}\left(Z_{i}\right)^{1 / 2} G\left(Z_{i}\right)-V_{T}\right)^{2}
\end{aligned}
$$

where the constant $V_{T}$ is a guess of the option value. Indeed, the minimization of (24) is equivalent to the one of the real variance of the estimator (21) as

$$
S_{2}(\theta)=\tilde{\Sigma}_{\theta}^{2}+\left(E_{P}[G(Z)]-V_{T}\right)^{2} .
$$

The algorithm for the determination of the optimal sampling distribution within a certain trial family can be therefore summarized as it follows:

- Generate a suitable number $N_{p}^{\prime}$ of replications of the state variables $Z$ according to the original probability distribution $P(Z)$;

- Choose a trial probability distribution $\tilde{P}_{\theta}(Z)$, and an initial value of the vector of parameters $\theta$;

- Set $x_{i} \rightarrow Z_{i}, f_{\theta}(x) \rightarrow W_{\theta}(Z)^{1 / 2} G(Z)$ and $y_{i} \rightarrow 0$ (resp. $y_{i} \rightarrow V_{T}$ ) and call a least squares fitter, say LSQ $\left[x, y, f_{\theta}(X), \theta\right]$, providing the optimal $\theta=\theta^{\star}$ by minimizing the second moment of the estimator $m_{2}(\theta)$, Eq. (23) $\left[\right.$ resp. $S_{2}(\theta)$, Eq. (24)].

Once the optimal parameters $\theta^{\star}$ have been determined through the least squares algorithm, one can perform an ordinary Monte Carlo simulation by sampling the probability distribution $\tilde{P}_{\theta \star}(Z)$, and calculating expectation values according to Eq. (77).

As it will be illustrated with the numerical examples of Sec. $\nabla$ this procedure does not add a significant overhead to the simulation, because just a relatively small number of replications $N_{p}^{\prime} \ll N_{p}$ is usually required to determine the optimal parameter $\tilde{\theta}^{\star}$. This is due to the fact that the configurations over which the optimization is performed are fixed. As a result of this form of correlated sampling [12], the difference in the $m_{2}(\theta)$ 's for two sets of values of the parameters being optimized is much more accurately determined than the values of the $m_{2}(\theta)$ 's themselves. This rather surprising feature is rooted in the fact that the minimization of Eq. (23) as a means to optimize the trail density, $\tilde{P}_{\theta}(Z)$, can be justified in terms of a genuine maximum likelihood criteria [18], and it is therefore

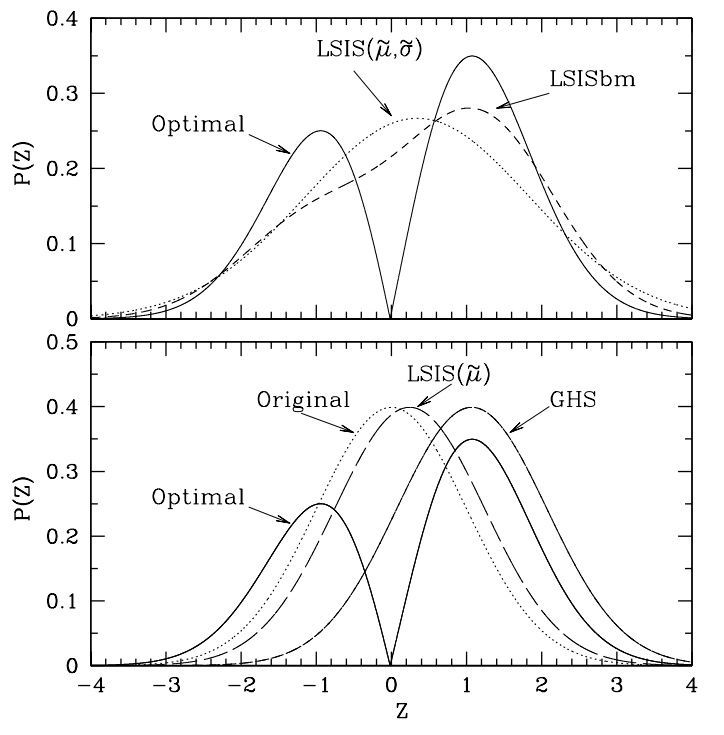

FIG. 3: Sampling probability density functions for a straddle option (30) with $r=0.05, X_{0}=K=50, \sigma=0.3, T=1$, as obtained with LSIS [optimizing just the drift, $\operatorname{LSIS}(\tilde{\mu})$, both the drift and the volatility, $\operatorname{LSIS}(\tilde{\mu}, \tilde{\sigma})$, and using the bi-modal trial density (31), LSISbm], and the saddle point approximation (GHS). The original (13) and the optimal (9) sampling densities also shown for comparison.

independent on how accurately $m_{2}(\theta)$ approximates the quantity (22).

Clearly, the fact that only a limited number of Monte Carlo samples is required for the optimization of the trial density limits the overhead introduced by the LSIS algorithm. This makes LSIS a practical strategy for variance reduction.

In the following we will demonstrate the effectiveness of this simple strategy by applying it to a variety of test cases, and we will compare the present approach with the ones most recently proposed in the literature [6, 8, ,9, 10].

\section{NUMERICAL EXAMPLES}

\section{A. European Options}

We will start by considering European style options. These are the simplest possible examples of financial relevance that allow one to illustrate the LSIS strategy. In particular, we will consider first standard Call and Put options, written on a single underlying asset, $X(t)$, following the $\log$ normal process (14). Here $N=M=$ $L=1$, and $\Delta t_{0}=T$. The payout function reads as in Eq. (15) with $\bar{X} \rightarrow X_{1} \equiv X(T), \Phi(X)=(X-K)^{+}$and $\Phi(X)=(K-X)^{+}$for the Call and the Put, respectively, and $K$ is the strike price.

In these cases, the probability distribution $P(Z)$ is a univariate Gaussian distribution, and the option value (11) involves the integration of a function of a single vari- 
able. As discussed in Sec. III, Importance Sampling techniques seek a sampling probability density $\tilde{P}_{\theta}(Z)$ as close as possible to the optimal sampling distribution, Eq. (9) (see Figure 1). The simplest choice for $\tilde{P}_{\theta}(Z)$, in this setting, is a Gaussian distribution of the form (16) (with $d=1$ ), so that the only parameter $\theta$ to optimize is the $\operatorname{drift} \tilde{\mu}$.

The LSIS algorithm described in Sec. IV is particularly straightforward to implement, and involves a presimulation stage in which a set of $N_{p}^{\prime}$ standard normal random numbers are generated and stored, and a least squares optimization routine is invoked. As anticipated, we found that the least squares fitter was able to determine successfully the optimal $\tilde{\mu}$ with as little as $N_{p}^{\prime} \simeq 50$ Monte Carlo replications, thus making the approach useful in practice.

In Tables II and II we compare the results obtained with LSIS with the ones obtained by means of the Robbins Monro (RM) adaptive Monte Carlo (as quoted in Ref. [10]), and the saddle point approach of GHS [6]. Here, as an indicator of the efficiency gains introduced by the different strategies of Importance Sampling, we have defined the variance ratio as

$$
\mathrm{VR}=\left(\frac{\sigma(\text { Crude } \mathrm{MC})}{\sigma(\mathrm{IS})}\right)^{2}
$$

where the numerator and denominator are the statistical errors (for the same number of Monte Carlo paths) of the Crude and the Importance Sampling estimators, respectively. In particular, for this and the following examples, we have estimated the uncertainties on the statistical errors, and on the VR's by performing a standard error analysis of the outcome of several Monte Carlo simulations for different random number seeds.

We found that the different methods produce a significative and comparable variance reduction. In particular, as it is intuitive, the change of drift is more effective for low volatility, and deep in and out of the money options. In this case, LSIS and GHS optimized trial distributions $\tilde{P}_{\bar{\mu}}(Z)$ are very similar as shown Fig. 1. This could be expected as, in this case, the optimal Importance Sampling distribution (9) can be effectively approximated by a Gaussian with the same mode and variance, so that the GHS approach produces accurate results.

However, the LSIS method is not limited to Importance Sampling strategies based on a pure change of drift, and one can easily introduce additional optimization parameters in the trial density. For instance, in this example it makes sense to introduce the sampling volatility, $\tilde{\sigma}$,

$$
\tilde{P}_{\tilde{\mu}, \tilde{\sigma}}(Z)=\left(2 \pi \tilde{\sigma}^{2}\right)^{-1 / 2} e^{-(Z-\tilde{\mu})^{2} / 2 \tilde{\sigma}^{2}} .
$$

As illustrated in Fig. 1, by adjusting both $\tilde{\mu}$ and $\tilde{\sigma}$, one obtains a trial density closer to the optimal one. This corresponds to an additional variance reduction up to over one order of magnitude, as shown in Tables [ and [II.
Another simple example in which the ability to adjust the width of the sampling distribution proves effective is the Butterfly spread option,

$$
\Phi(X)=\left(X-K_{1}\right)^{+}-2\left(X-K_{2}\right)^{-}+\left(X-K_{3}\right)^{+},
$$

with $K_{1}<K_{2}<K_{3}$. In this case, a pure change of drift can only force the expected end point of the sampled paths $X(T)$ to fall in the money, $K_{1}<X(T)<K_{3}$. However, for values of the volatility or the maturity large enough, a significative fraction of the sampled paths will still results in a zero payout. Hence, a pure change of drift is not very effective in reducing the variance. This is illustrated in Table III. Instead, by quenching the sampled volatility one can increase the number of paths falling in the money thus reducing the variance. For instance, in the example of Table III. for $S_{0}=50$ the saddle point change of drift is very small, and does not alter the variance. Instead, by allowing the sampling volatility to vary, we get $\tilde{\mu}^{\star} \simeq-0.02$ and $\tilde{\sigma}^{\star} \simeq 0.14$, reducing the variance by more than two orders of magnitude.

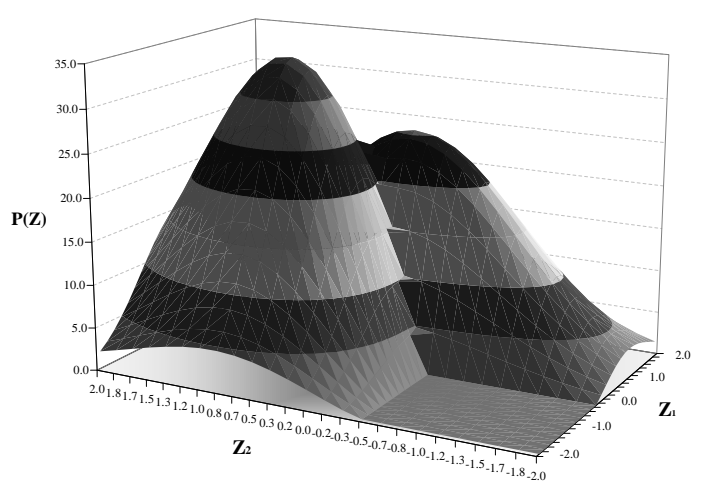

FIG. 4: Optimal sampling distribution, Eq. (9), for the European basket Call option (32) for $r=0.05, K=100, X_{1}^{0}=100$, $X_{2}^{0}=105, \sigma_{1}=\sigma_{2}=0.3, T=1$.

\section{B. Asian Call option}

As a second example we consider here the single underlying $(L=1)$ arithmetic Asian option Eq. (15), with a Call payout, $\Phi(Z)=e^{-r T}(\bar{X}-K)^{+}$, and $M$ observation dates.

In this case, the calculation of the expectation value (11) involves a multi-dimensional integral over the probability distribution (13). However, the implementation of LSIS is not more difficult than for the European payout. Indeed, the fact that the sample points $Z_{i}$ are in the present case $M$-dimensional vectors is practically irrelevant for the LSIS procedure. All the least square fitter needs is a method that, given a configuration $Z_{i}$ and the vector $\theta$, provides the value of the target function $f_{\theta}\left(Z_{i}\right)=W_{\theta}\left(Z_{i}\right)^{1 / 2} G\left(Z_{i}\right)$. 
TABLE VI: Comparison between LSIS, the adaptive Robbins-Monro (RM) method (as quoted in Ref. [10]), for the price of an Asian Call option (15) on a lognormal asset (14) for different values of the volatility $\sigma$, and of the strike price $K$. The parameters used are $r=0.1, X_{0}=50, T=0.5$, and the number of simulated paths is 1,000,000 for Crude MC and LSIS, 800,000 for RM. The uncertainties on the RM variance reductions are rough estimates based on the number of simulated paths.

\begin{tabular}{|c|c|c|c|c|c|c|c|}
\hline \multirow[b]{2}{*}{$M$} & \multirow[b]{2}{*}{$\sigma$} & \multirow[b]{2}{*}{$K$} & \multirow{2}{*}{$\begin{array}{l}\text { Crude MC } \\
\text { Price }\end{array}$} & \multicolumn{2}{|c|}{ LSIS } & \multicolumn{2}{|c|}{$\mathrm{RM}$} \\
\hline & & & & Price & VR & Price & VR \\
\hline \multirow[t]{6}{*}{20} & 0.1 & 40 & $10.7820(21)$ & $10.78243(22)$ & $91(2)$ & 10.73 & $58(2)$ \\
\hline & & 50 & $1.6045(16)$ & $1.60398(61)$ & $6.9(1)$ & 1.53 & $7.0(1)$ \\
\hline & & 55 & $0.04919(29)$ & $0.049204(54)$ & $28.8(5)$ & 0.037 & $6.0(5)$ \\
\hline & 0.3 & 40 & $10.8284(62)$ & $10.8277(17)$ & $13(1)$ & 10.77 & $14(1)$ \\
\hline & & 50 & $3.1373(44)$ & $3.1352(15)$ & $8.6(1)$ & 2.99 & $9.0(1)$ \\
\hline & & 60 & $0.3790(16)$ & $0.37861(35)$ & $20.9(5)$ & 0.320 & $28.0(5)$ \\
\hline
\end{tabular}

TABLE VII: Comparison between LSIS, the adaptive approach of Su and Fu (SF) (as quoted in Ref. [8]), for the price of a partial average Asian Call option (15) and (29) on a lognormal asset (14) for different values of the volatility $\sigma$, and of the strike price $K$. The parameters used are $r=0.05, X_{0}=100, T=1.0, M=365, M_{0}=305$, and the number of simulated paths is 1,000,000 for Crude MC, and LSIS, and 50,000 for SF. The uncertainties on the SF variance reductions are rough estimates based on the number of simulated paths.

\begin{tabular}{|c|c|c|c|c|c|c|}
\hline \multirow[b]{2}{*}{$\sigma$} & \multirow[b]{2}{*}{$K$} & \multirow{2}{*}{$\begin{array}{c}\text { Crude MC } \\
\text { Price }\end{array}$} & \multicolumn{2}{|c|}{ LSIS } & \multicolumn{2}{|c|}{$\mathrm{SF}$} \\
\hline & & & Price & VR & Price & VR \\
\hline \multirow[t]{8}{*}{0.2} & 100 & $9.792(14)$ & $9.7816(46)$ & $9.3(1)$ & 9.747 & $6.7(3)$ \\
\hline & 110 & $5.413(11)$ & $5.4256(33)$ & 11.1(1) & 5.397 & $8.2(3)$ \\
\hline & 120 & $2.7758(77)$ & $2.7669(20)$ & $14.8(2)$ & 2.730 & $11.0(6)$ \\
\hline & 130 & $1.3038(52)$ & $1.3096(12)$ & $18.8(1)$ & 1.284 & $17.0(4)$ \\
\hline & 140 & $0.5861(35)$ & $0.58298(61)$ & $33(1)$ & 0.575 & $25(3)$ \\
\hline & 150 & $0.2421(22)$ & $0.24592(30)$ & $54(4)$ & 0.241 & $44(12)$ \\
\hline & 160 & $0.1003(14)$ & $0.09973(14)$ & $100(8)$ & 0.0980 & $85(20)$ \\
\hline & 170 & $0.03761(84)$ & $0.039000(64)$ & $172(20)$ & 0.0380 & $173(80)$ \\
\hline \multirow[t]{8}{*}{0.3} & 100 & $13.323(21)$ & $13.3527(65)$ & $10.4(5)$ & 13.295 & $7(1)$ \\
\hline & 110 & $9.182(18)$ & $9.1564(54)$ & $11.1(5)$ & 9.103 & $8(1)$ \\
\hline & 120 & $6.1041(15)$ & $6.1258(39)$ & $14.8(5)$ & 6.059 & $10(1)$ \\
\hline & 130 & $4.020(12)$ & $4.0034(31)$ & $15.0(5)$ & 3.985 & $12(3)$ \\
\hline & 140 & $2.5624(98)$ & $2.5769(23)$ & $18.1(7)$ & 2.556 & $15(2)$ \\
\hline & 150 & $1.6410(80)$ & $1.6325(13)$ & $38(1)$ & 1.603 & $22(2)$ \\
\hline & 160 & $1.0143(62)$ & $1.02355(90)$ & $47(2)$ & 1.006 & $30(5)$ \\
\hline & 170 & $0.6408(50)$ & $0.63681(61)$ & $67(3)$ & 0.623 & $36(6)$ \\
\hline
\end{tabular}

In order to compare LSIS with the other approaches proposed in the literature we have considered a Gaussian trial density of the form Eq. (16). Here such density depends on as many parameters, $\tilde{\mu}=\left(\tilde{\mu}_{0}, \ldots, \tilde{\mu}_{M-1}\right)$, as are the sampling dates in the Euler discretization of the process (12). A typical outcome of the optimized drift vector produced by the LSIS procedure is shown in Fig. 2.

\section{Comparison with the Saddle Point Approximation}

As shown in Table IV] LSIS provides a very effective variance reduction for the Asian Call options considered, similar to the one obtained with the approach of GHS. This could be expected on general grounds as in the case of the European Call and Put options. Indeed, it is not difficult to verify that the single asset Asian Call option generates a single-mode optimal sampling den- sity $P_{\text {opt }}(Z) \propto G(Z) P(Z)$, with covariance close to one. Hence, as discussed in the previous Section, the optimal sampling density can be accurately represented by a Gaussian distribution, and the saddle point method provides an accurate approximation of the optimal drift vector. Indeed, as shown Fig. 2, the optimal drift vectors obtained with the LSIS and the GHS approaches are quite similar, with an overlap of $\sim 0.99$ when both normalized to one.

As anticipated, we found that a few hundreds of Monte Carlo configurations and 10-20 iterations of the least squares fitter, were typically enough to determine the optimal drift vector in (16) for $M \simeq 50$, thus making the overhead of the pre-simulation stage rather limited. However, as the number of time steps - or more in general the number of components of the drift vector - increases, the complexity of the optimization problem increases as well. Nevertheless, as suggested in Ref. [7], one can significantly reduce the computation time associated with the optimization stage by approximating the 
TABLE VIII: Importance Sampling results for the European style straddle (30) obtained by means of the saddle point approximation (GHS) and LSIS, with the optimization of the drift $[\operatorname{LSIS}(\tilde{\mu})]$, and the drift and the volatility $[\operatorname{LSIS}(\tilde{\mu}, \tilde{\sigma})]$. The parameters used are $r=0.05, X_{0}=K=50, \sigma=0.3, T=1$, and correspond to the probability distributions sketched in Fig. 3 . The number of simulated paths is $1,000,000$.

\begin{tabular}{|c|c|c|c|c|c|c|}
\hline \multirow{2}{*}{$\begin{array}{c}\text { Crude MC } \\
\text { Price }\end{array}$} & \multicolumn{2}{|c|}{ GHS } & \multicolumn{2}{|c|}{$\operatorname{LSIS}(\tilde{\mu})$} & \multicolumn{2}{|c|}{$\operatorname{LSIS}(\tilde{\mu}, \tilde{\sigma})$} \\
\hline & Price & VR & Price & VR & Price & VR \\
\hline $11.803(10)$ & $11.800(33)$ & $0.10(1)$ & $11.8038(88)$ & $1.30(1)$ & $11.8047(55)$ & $3.00(2)$ \\
\hline
\end{tabular}

drift vector with a continuous function parameterized by a small number of parameters. These are in turn tuned by the least square algorithm in order to determine an approximate optimal drift vector. We have found that a particularly effective realization of this approach is to approximate the drift vector by a piecewise linear function, parameterized by its values where it changes slope (the so-called knot points). For instance, in the present examples, optimizing over 4 to 8 equally spaced knot points provides variance reductions practically indistinguishable from the ones obtained by a full optimization. In addition, the drift vector generally changes continuously with the market parameters. As a result, an additional computational saving can be obtained by starting the presimulation from a drift vector optimized for a case with a similar set of parameters.

As anticipated in Sec. II A Importance Sampling can be also naturally combined with Stratified Sampling by choosing the optimal drift vector as the stratification direction (for further details see Ref.[6]). This, as shown in Table $\mathrm{V}$, gives rise to a further reduction of the variance, which can be of several order of magnitudes, depending on the option parameters, thus resulting in quite remarkable savings in computational time.

\section{Comparison with Adaptive Monte Carlo approaches}

It is also interesting to compare the LSIS results with those obtained by means of the recently proposed adaptive Monte Carlo methods briefly discussed in Sec. IIA. As illustrated in Table VI we found that our approach achieves variance reductions similar to the ones obtained with the Robbins-Monro algorithm of Ref.[10]. However, for the example considered, LSIS appears to perform better for small values of the volatility and deep in and out of the money options. This is particularly remarkable when considering that the pre-simulation stage required by the Robbins-Monro algorithm involves sampling a number of Monte Carlo paths much larger than that required by the LSIS approach. For instance, in the present example, the number of iterations quoted in Ref. [10] is 400,000 while the LSIS results were obtained by sampling just $N_{p}^{\prime} \simeq 400$ configurations. In addition, LSIS does not have any exogenous parameter to be fine tuned in order to achieve an efficient optimization, thus making the approach easier to be automated.
Finally, in order to compare with the adaptive Monte Carlo approach of $\mathrm{Su}$ and $\mathrm{Fu}$ [8, 9], we have slightly modified the Asian option example and considered a payout depending on a partial average

$$
\bar{X}=\frac{1}{M-M_{0}} \sum_{i=M_{0}}^{M} X_{i}
$$

where $M_{0}$ is the time step index corresponding to the first observation. The results we have obtained in this case are reported in TableVII, and show that LSIS generally outperforms the approach of Ref. [8, 9]. This is expected as LSIS involves a more general class of sampling distribution (16) as the change of drift is not constrained to be uniform along the simulation path.

\section{Multi-modal Examples}

All the examples considered in the previous Sections were characterized by a single mode optimal sampling distribution (91). In these cases, an Importance Sampling strategy based on the Gaussian trial distributions (16) or (27) provides good results. In this Section we will consider instead option pricing problems that are characterized by multi-modal optimal sampling densities. These are very common in practice, and typically arise if the payout function $G(Z)$ is not monotonic, or for claims written on multiple assets. We will show that in these cases a simple Importance Sampling strategy based on a pure change of drift of a Gaussian density proves ineffective. In contrast, LSIS allows one to work with trial densities tailored to the problem at hand, including multimodal distributions. As in Section VA we begin by illustrating the effectiveness of LSIS with a simple European style payout.

\section{Straddle}

A simple example of a pricing problem characterized by a multi-modal sampling density is the European straddle:

$$
\Phi(X)=(X-K)^{+}+(K-X)^{+} .
$$

As illustrated in Fig. 3, the corresponding optimal sampling distribution, $P_{\text {opt }}(Z) \propto G(Z) \exp \left(-Z^{2} / 2\right)$, has two 
well separated maxima. As a result, saddle point approaches based on a Gaussian distribution, e.g., centered on the higher of the two modes, provide a poor approximation of the optimal sampling density. Indeed, as shown in TableVIII, the saddle point method increases the variance with respect to the crude Monte Carlo estimator. On the contrary, LSIS based on the simple trial density (16) provides a small shift of the drift towards the higher mode of the optimal distribution [Fig. 3]. This can be easily understood. In fact, the best way to approximate a bi-modal distribution by means of a Gaussian density is to center the latter around the center of mass of the former, in this case $\tilde{\mu} \simeq 0.24$. Yet, since the two maxima of the optimal distribution are well separated, its overlap with the trial density is still rather poor. As a result, LSIS based on a trial density of the form (16) produces only a small variance reduction. Better results can be obtained by adjusting the width of the trial density. Indeed, as also illustrated in Fig. 3, by increasing the standard deviation of the sampling density one achieves a better approximation of the optimal distribution. This leads to a sizable reduction of the variance of a factor of 3 .

An even better ansatz for the optimal density is clearly represented by a bi-modal trial density of the form

$$
\tilde{P}(Z)=(2 \pi)^{-d / 2}\left[w_{a} e^{-\left(Z-\mu_{a}\right)^{2}}+w_{b} e^{-\left(Z-\mu_{b}\right)^{2}}\right],
$$

where $w_{a}+w_{b}=1$ (and $d=1$ in this specific case) that can be optimized over $\mu_{a}, \mu_{b}$, and $w_{a}$. The simulation of a density of this form is straightforward as it simply implies choosing on each Monte Carlo step one of the two Gaussian components in (31), and sample a configuration $Z_{i}$ according to it. This can be done by extracting an auxiliary uniform random number $\xi \in[0,1]$, and extracting $Z_{i}$ according to the first Gaussian component if $\xi<w_{a}$, and according to the second otherwise. The optimized distribution obtained using this bi-modal trial density is sketched in Fig. 3. and corresponds to a further sizable reduction of the variance (Table VIII). Remarkably, the same form of sampling density (31) can be used also for Asian style straddles (here $d=M, \mu_{a}$ and $\mu_{b}$ are $M$-dimensional vectors), and produces a very effective variance reduction, as illustrated in Table IX.

TABLE IX: Variance Reduction obtained with LSIS for a European $(M=1)$, and Asian style ( $M=16$ and 64$)$ straddle (30) using the bi-modal sampling distribution (31). The parameters used are $r=0.05, X_{0}=K=50, \sigma=0.3$, $T=1$, and correspond for $M=1$ to the probability distributions sketched in Fig. 3. The number of simulated paths is $1,000,000$.

\begin{tabular}{cccc}
\hline \hline & Crude MC & \multicolumn{2}{c}{ LSIS } \\
$M$ & Price & Price & VR \\
\hline 1 & $11.803(10)$ & $11.8009(44)$ & $5.17(5)$ \\
16 & $7.0604(58)$ & $7.0559(26)$ & $4.98(5)$ \\
64 & $6.8200(55)$ & $6.8163(26)$ & $4.47(5)$ \\
\hline \hline
\end{tabular}

TABLE X: Importance Sampling results for the Asian style basket option (32) obtained by means of LSIS, using the bimodal sampling distribution (31). The parameters used are $r=0.05, T=1, X_{0}^{(1)}=100, X_{0}^{(2)}=105, \sigma_{1}=0.3, \sigma_{2}=0.3$, and correspond (for $K=100$ and $M=1$ ) to the probability distributions sketched in Fig. 4. The number of simulated paths is 500,000 .

\begin{tabular}{ccccc}
\hline \hline & & Crude MC & \multicolumn{2}{c}{ LSIS } \\
$M$ & $K$ & Price & Price & VR \\
\hline 1 & 90 & $34.555(41)$ & $34.587(14)$ & $8.58(7)$ \\
& 100 & $26.621(39)$ & $26.589(15)$ & $6.76(3)$ \\
& 110 & $19.749(36)$ & $19.776(13)$ & $7.7(1)$ \\
16 & 90 & $24.876(24)$ & $24.8700(87)$ & $7.6(1)$ \\
& 100 & $16.428(22)$ & $16.4081(81)$ & $7.4(1)$ \\
& 110 & $9.711(19)$ & $9.6918(68)$ & $7.8(1)$ \\
32 & 90 & $24.537(23)$ & $24.5539(84)$ & $7.50(5)$ \\
& 100 & $16.082(22)$ & $16.0734(82)$ & $7.2(1)$ \\
& 110 & $9.381(18)$ & $9.3752(68)$ & $7.0(1)$ \\
\hline \hline
\end{tabular}

\section{Basket Call Options}

Basket options are another very common class of contingent claims that can give rise to multi-modal optimal distributions. This is illustrated in Fig. 4 for a very simple European style Call option on the maximum of $L=2$ underlying assets following the process (14)

$$
\Phi(X)=\left(\max \left(X_{1}, X_{2}\right)-K\right)^{+} .
$$

Also in this case, LSIS based on a bi-modal trial density of the form (31) provides a significative variance reduction that persists when introducing Asian features in the payout (see Table $[\mathrm{X}$ ). We obtained similar results for other multi assets options by generalizing the form of the trial density (31).

\section{CONCLUSIONS}

In this paper we have described a simple Importance Sampling technique based on a least squares minimization of straightforward implementation. The resulting strategy, dubbed Least Square Importance Sampling (LSIS), provides an effective variance reduction technique that lends itself to a variety of applications.

We have presented several numerical examples in a diffusive setting, and we have shown that LSIS, when restricted to the optimization of the drift of a diffusion process, provides variance reductions similar to those obtained with existing approaches [6, 8, 9, 10]. However, LSIS is not limited to the determination of the optimal mean of a Gaussian sampling distribution. As a result, it outperforms other approaches when the ability to adjust the width of such distribution, or to sample non-Gaussian and multi-modal densities, is important to achieve variance reductions.

The LSIS strategy can be applied to any Monte Carlo setting provided that a reasonable ansatz for the opti- 
mal sampling distribution is available. This makes LSIS a flexible Importance Sampling approach, that can be used across a variety of financial applications, ranging from Value at Risk (VaR) estimation, to portfolio credit risk management. This is currently the object of further investigations. discussions, and Paul Glasserman for an enlightening lecture that inspired this work. The opinion and views expressed in this paper are uniquely those of the author, and do not necessarily represent those of Credit Suisse Group.

\section{Acknowledgments}

It is a pleasure to acknowledge Gabriele Cipriani, David Shorthouse, and Mark Stedman for stimulating

[1] P. Glasserman, Monte Carlo Methods in Financial Engineering (Springer, New York, 2004).

[2] J. C. Hull, Options, Futures and Other Derivatives (Prentice Hall, New Jersey, 2002).

[3] R. Reider (1993), working paper.

[4] P. Boyle, M. Broadie, and P. Glasserman, Journal of Economic Dynamics and Control 21, 1257 (1997).

[5] F. Vazquez-Abad and D. Dufresne, in Proceedings of 1998 Winter Simulation Conference, edited by D. Medeiros, E. Watson, J. Carson, and M. Manivann (IEEE Press, Washington DC, 1998), pp. 1493-1500.

[6] P. Glasserman, P. Heidelberger, and P. Shahabuddin, Mathematical Finance 9, 117 (1999).

[7] P. Glasserman, P. Heidelberger, and P. Shahabuddin, Journal of Derivatives 7, 32 (1999).

[8] Y. Su and M. C. Fu, in Proceedings of 2000 Winter Simulation Conference, edited by J. Joines, R. Barton, K. Kang, and P. Fishwick (IEEE Press, Piscataway NJ, 2000), pp. 587-596.

[9] Y. Su and M. C. Fu, Journal of Computational Finance 5, 27 (2002).

[10] B. Arouna, Journal of Computational Finance 7, 1245 (2003).

[11] P. Glasserman and J. Li, Management Science 51, 1463 (2005).

[12] C. J. Umrigar, K. G. Wilson, and W. Wilkins, Physical Review Letters 60, 1719 (1988).

[13] J. Harrison and D. Kreps, Journal of Economic Theory 20, 381 (1979).

[14] M. Musiela and M. Rutkowski, Martingale Methods in Financial Modelling (Springer, New York, 2002).

[15] M. Baxter and A. Rennie, Financial Calculus: An In- troduction to Derivative Pricing (Cambridge University Press, Cambridge, 1996).

[16] O. Kallenberg, Foundations of Modern Probability (Springer, New York, 1997).

[17] W. H. Press, S. A. Teukolsky, W. T. Vetterling, and B. P. Flannery, Numerical recipes in $C++$ : the art of scientific computing (Cambridge University Press, Cambridge, 2002).

[18] D. Bressanini, G. Morosi, and M. Mella, Journal Chemical Physics 116, 5345 (2002).

[19] In the present discussion we will treat the $Z_{i}$ 's as continuous variables, however all the results also apply if some or all of them can assume only a discrete set of values. For any of such variable, the symbol $\int d Z_{i}$ is to be interpreted as a sum over the possible outcomes.

[20] In particular, the Monte Carlo integration becomes unfeasible if the variance of the estimator diverges, giving rise to the so-called sign-problem instability. Although this problem is the crux of Monte Carlo simulations in several branches of the Physical Sciences, see, e.g., S. Sorella and L. Capriotti, Physical Review B 61, 2599 (2000), this issue does not usually affect financial contexts.

[21] It is possible to show [17] that, when $G(Z)$ does not have a definite sign, the optimal sampling density has the similar form $P_{o p t}=|G(Z)| P(Z) / V$, although in this case the optimal variance is not zero.

[22] The use of other discretization schemes does not alter the present discussion.

[23] B. Arouna, private communication. 